Instituto Internacional de Investigación y Desarrollo Tecnológico Educativo INDTEC, C.A.

DOI: https://doi.org/10.29394/Scientific.issn.2542-2987.2020.5.17.6.122-142

OAI-PMH: http://www.indteca.com/ojs/index.php/Revista Scientific/oai

Artículo Original / Original Article

\title{
Desarrollo de competencias didácticas docentes para la enseñanza de nociones histórico-culturales en Educación Inicial
}

\author{
Autora: Laury Dayana Mejías Escorcha \\ Universidad Pedagógica Experimental Libertador, UPEL \\ laurymejias84@gmail.com \\ Barinas, Venezuela \\ https://orcid.org/0000-0003-2749-4621
}

\section{Resumen}

El presente estudio busca determinar la importancia del desarrollo de competencias didácticas docentes para la enseñanza de nociones históricoculturales en los niños y niñas en Educación Inicial, en atención al componente curricular Historia local, regional y nacional, en la ciudad de Barinas, estado Barinas. Fue seleccionado el enfoque de investigación cuantitativa con diseño descriptivo, utilizando procesos inductivos para explorar y describir; lo cual reflejará una realidad específica al caracterizar, registrar y analizar; bajo la modalidad de proyecto factible, promoviendo estrategias que permitan el surgimiento de soluciones a la problemática. Se aplica cuestionario de diez (10) ítems a una muestra de diez (10) docentes del Subsistema de Educación Inicial, Etapa Preescolar (de 4 años, 10 meses a 5 años, 10 meses) en 05 Centros de Educación Inicial de la ciudad de Barinas. Se evidencia como resultado la carencia de metodología didáctica docente que apoye la reflexión para la enseñanza de la historia y la cultura, por lo cual se prevé el diseño de estrategias didácticas que generen medios de construcción del pensamiento histórico-cultural en los niños y niñas basados en la reflexión continua, para que las celebraciones cívicas y efemérides les sean significativas y valoren positivamente su entorno.

Palabras clave: educación; historia; cultura; mediación.

Cómo citar este artículo:

Mejías, L. (2020). Desarrollo de competencias didácticas docentes para la enseñanza de nociones histórico-culturales en Educación Inicial. Revista Scientific, 5(17), 122-142, e-ISSN: 2542-2987. Recuperado de: https://doi.org/10.29394/Scientific.issn.2542-2987.2020.5.17.6.122-142

Fecha de Recepción: 02-12-2019
Fecha de Aceptación: 30-04-2020
Fecha de Publicación: 05-08-2020 
OAl-PMH: http://www.indteca.com/ojs/index.php/Revista_Scientific/oai

Artículo Original / Original Article

\title{
Development of teaching didactic competences for the teaching of
} historical-cultural notions in Initial Education

\begin{abstract}
The present study seeks to determine the importance of the development of teaching didactic competences for the teaching of historicalcultural notions in boys and girls in Initial Education, in attention to the curricular component Local, regional and national history, in the city of Barinas, Barinas state. The quantitative research approach with descriptive design was selected, using inductive processes to explore and describe; which will reflect a specific reality when characterizing, registering and analyzing; under the feasible project modality, promoting strategies that allow the emergence of solutions to the problem. A questionnaire of ten (10) items is applied to a sample of ten (10) teachers of the Initial Education Subsystem, Preschool Stage (from 4 years, 10 months to 5 years, 10 months) in 05 Initial Education Centers of the city of Barinas. As a result, the lack of teaching didactic methodology that supports reflection for the teaching of history and culture is evident, for which reason the design of didactic strategies that generate means of construction of historical-cultural thinking in children based on in continuous reflection, so that civic celebrations and ephemeris are meaningful to them and positively value their environment.
\end{abstract}

Keywords: education; history; culture; mediation.

How to cite this article:

Mejías, L. (2020). Development of teaching didactic competences for the teaching of historicalcultural notions in Initial Education. Revista Scientific, 5(17), 122-142, e-ISSN: 2542-2987. Recovered from: https://doi.org/10.29394/Scientific.issn.2542-2987.2020.5.17.6.122-142

Date Received: 02-12-2019
Date Acceptance:

30-04-2020
Date Publication:

05-08-2020 


\section{Introducción}

Los niños y niñas asocian lo memorístico y acumulativo según lo que aprenden por medio de los sentidos, de lo que perciben en diferentes contextos, al jugar solos o en grupos experimentando situaciones cotidianas y adquiriendo hábitos. A partir de una intervención docente eficiente, el niño y la niña serán capaces de generar respuestas a estos estímulos, lo cual se traduce como aprendizaje significativo a la par de sus propios procesos de internalización.

Es decir, para el niño o niña de este nivel es común dar a las cosas su propio estilo narrativo, los acontecimientos los relaciona con su propia historia, personal o comunitaria, asociando procesos cognitivos/sensoriales presentes en su conocimiento con lo que observa y memoriza; por ello ha de estimulárseles el interés a partir de la enseñanza con múltiples referencias a las cosas concretas más que a sus descripciones verbales; por lo tanto tienen derecho y deber de adquirir valores que posibiliten el conocimiento y apropiación de su entorno, preservando sus tradiciones y costumbres como componentes de apreciación histórica y cultural.

Se tiene entonces que enseñar Historia y cultura en el contexto de Educación Inicial es un proceso regresivo que debe abordarse no como un conocimiento adicional, sino como una reflexión permanente, favoreciendo la curiosidad básica en los niños y niñas que los lleve a preguntarse cómo se hizo el presente e indagar, partiendo de ese presente hacia el pasado indagando curiosamente más allá de lo que puedan apreciar a simple vista: el propio, el de sus padres o abuelos, el de su comunidad; lo que le llevará, a corto y mediano plazo, a preguntarse sobre un pasado más lejano.

Por ello, se hace necesario cuestionarse; ¿EI discurso historiográfico en el niño y niña del Subsistema de Educación Inicial (Etapa Preescolar) puede ser significativo si no es vivencial?. Al respecto, García (2015), señala que: "[...] para que los niños y niñas comprendan mejor los sucesos 
históricos, se debe dar importancia al desarrollo de habilidades y conocimientos a través de las nociones de espacio, tiempo y cambio histórico [...]" (pág. 53). En teoría, según lo establecido por el Ministerio del Poder Popular para la Educación (2007a), en el documento Subsistema de Educación Inicial Bolivariana: Currículo y Orientaciones Metodológicas:

[...] Una persona poseedora y promotora de una conducta ética, moral, social, cultural y cónsona con los valores locales, regionales y de la nacionalidad venezolana; así como de habilidades para comunicarse con el colectivo comunitario, en tanto posee una sólida conciencia [...] (pág. 17).

Es decir, la metodología didáctica para la enseñanza de la Historia y la cultura, debe contar en la práctica del docente de Educación Inicial, y en función de ello, debe ser capaz de diseñar estrategias para que los niños y niñas adquieran nociones sobre nacionalismo, patriotismo, amor y orgullo por su localidad, costumbres, tradiciones y valores; siguiendo la perspectiva del modelo constructivista y estructuralista de la Educación Bolivariana.

Suele ser comentario común entre las docentes de Educación Inicial que es difícil enseñar y aprender Historia y cultura significativamente, debido a la edad de los niños y niñas en esta etapa. El contenido histórico-cultural en Educación Inicial generalmente se aborda en el llamado lunes cívico, actividad destinada a resaltar las efemérides sobre hechos culturales y personajes históricos relevantes-, donde las docentes sólo brindan narraciones que son retazos de la realidad desvinculados de su origen, descontextualizados, donde no se evidencian el sentido de pertenencia por la cultura venezolana ni el proceso de apreciación de los hechos y personajes a los que se hace mención. Puede suponerse entonces que el valor educativo que el Centro de Educación Inicial Bolivariano (CEIB) da a la enseñanza de la Historia y la cultura, no está acorde con el rol que las docentes juegan en este proceso en relación a su práctica didáctica consciente. 
Para evidenciar lo anterior, la investigadora hace referencia al contexto de cinco (05) Centros de Educación Inicial en la ciudad de Barinas, estado Barinas: Centro de Educación Inicial Bolivariano (CEIB) "Cuatricentenario" en el Barrio El Molino; CEIB "María Larriva Salas" en la Urbanización "Cuatricentenaria"; el CEIB "Los Pericos", en la Urbanización José Antonio Páez; el CEIB "Los Retoñitos" en el sector Alto Barinas Norte y el CEIB "India Urquía en el Complejo Habitacional "Ciudad Tavacare". Durante el desarrollo del lunes cívico se pudo constatar, mediante la observación y la recopilación de datos anecdóticos, situaciones que representan las aristas del problema a estudiar, las cuales se detallan a continuación:

1. Durante el desarrollo de esta actividad frecuentemente el discurso de la docente se encuentra sobre el nivel de comprensión que poseen los niños y niñas de esta etapa, por el uso de términos y lenguaje corporal no adecuado.

2. En dicho discurso, sólo se indican datos para memorizar, no se apoya a los niños y niñas a ser reflexivos y críticos en las diferentes situaciones que se dan a su alrededor, evitando la internalización.

3. Se aborda a los niños y niñas en grupo grande en un espacio común (por lo general el patio central), donde la docente les relata el hecho histórico o se cita la biografía de un personaje dirigiéndose al grupo sin utilizar ningún tipo de dinámica mediadora ni material didáctico que cumpla este propósito.

4. En el aula se desarrolla una especie de socialización con los niños y niñas para que expliquen lo que entendieron, donde no se evidencia el uso de herramientas ni técnicas didácticas. Por ejemplo, se les indica que coloreen formatos, recibiendo sólo instrucciones precisas de la docente sobre aspectos de forma más que de fondo, actividad conductista que pretende ser didáctica pero que evidentemente los niños y niñas no alcanzan a comprender del todo.

5. No se utilizan instrumentos de evaluación para medir los alcances específicos según la finalidad prevista por el componente curricular. 
Lo anteriormente descrito es considerable, debido a que, si el conocimiento de la Historia y la cultura en el nivel Inicial se basa sólo en la memorización de datos inconexos, fuera de la lógica y comprensión, se les está iniciando a los niños y niñas en el sentimiento de aversión o por lo menos de aburrimiento que en muchas personas (docentes inclusive) causan la Historia y la cultura. Esto puede llevarlos a futuro a pensar en estas temáticas como el conocimiento de algo inútil y absurdo de memorizar y fácil de olvidar. La docente de Educación Inicial debe entonces buscar alternativas que permitan al niño y a la niña reflexionar para entender la Historia y la cultura de nuestro país a partir de la relación del pasado con el presente: un suceso cercano, lo propio, despertarles el interés por indagar en el pasado sobre lo actual, lo que los llevará a preguntarse sobre un pasado más lejano.

No se trata entonces de enseñar a los niños y niñas la Historia y la cultura en lecciones breves cada vez que se aborde la fecha marcada en el calendario escolar, sino que comiencen a construir su pensamiento históricocultural al reflexionar continuamente en su quehacer cotidiano y en participar con esa misma reflexión en las celebraciones cívicas y efemérides para que le sean significativas partiendo del presente al pasado, utilizando para ello medios didácticos que les permitan al niño y a la niña la interacción directa con el suceso que la docente desea que comprendan.

Dicha mediación didáctica debe comprender, aparte de la intervención orientadora de la docente, el uso de materiales como objetos, fotografías, anécdotas, música, entre otros; así como el testimonio de familiares y adultos significativos que compartan sus experiencias, historias de vida y de tradición oral. El componente docente ha de ser clave en este sentido, en acción y pensamiento, para que las actividades cívicas (históricas) y tradicionales (culturales) aborden aspectos diversos que permitan acceder a otros tipos de conocimientos, evitando además en lo posible que los niños y niñas festejen o conmemoren estas fechas significativas sin meditación alguna. 
Es por ello que la presente investigación pretende realizar un aporte científico al proporcionar a la docente de Educación Inicial un soporte complementario que le oriente al planificar acciones didácticas efectivas, afectivas y mediadoras para la enseñanza de la Historia y la cultura, reflejando rutinas para el desarrollo de la abstracción y la conceptualización, a la par del fomento de la identidad personal de los niños y niñas partiendo de valores sociales, mismos que llevarán consecuentemente al desarrollo de otros aspectos de su personalidad moral, entre ellos la identidad local y nacional, en función a lo previsto en el perfil del egresado y la egresada de este subsistema.

\section{Bases teóricas}

\subsection{Competencias docentes: hacia los objetivos de la enseñanza}

El desempeño profesional de un docente implica el desestimar el quehacer educativo que se realiza de forma monótona, automatizada. En la opinión de Salto y Cendón (2019), para el nivel de Educación Inicial, un desempeño profesional docente de calidad:

[...] Permite comprender el desarrollo integral del niño, puesto que en el nivel inicial los niños desarrollan todas sus habilidades de forma espiral, los ámbitos de aprendizaje, por lo que es necesario que las destrezas sean adquiridas a través de juegos y mediante la interrelación del niño con su entorno y pares sociales [...] (pág. 162).

Se hace indispensable entonces el desarrollo de competencias para el enriquecimiento de la praxis educativa cotidiana. En referencia al tema, Coronado (2013), define a las competencias docentes como: "[...] todos aquellos conocimientos, actitudes y habilidades que necesita un profesor para hallar respuesta satisfactoria a los conflictos y situaciones varias que puedan encontrarse en su contexto profesional [...]" (pág. 83). Es decir, es la interrelación de recursos entre lo teórico y lo práctico, entre lo que puede ser aplicado y lo que se aprende. Dichos recursos deben ser utilizados de manera 
eficaz por el docente para garantizar el aprendizaje de sus estudiantes en circunstancias específicas de su quehacer.

En el sentido de su aplicación, cada docente recibe una formación en competencias según el nivel donde enseñará, de forma que estén preparados para él, sin embargo, esta formación no es finita ni está exenta de equivocaciones. Partiendo del contexto y situaciones diversas que puedan presentarse, lo que se quiere es que las competencias se manifiesten a lo largo de la trayectoria profesional en función a la evolución del docente, según el interés que ponga en su formación continua y el provecho que obtenga del conocimiento que otorga la experiencia, a partir de lo cual podrá resolver problemas, revisar objetivamente su propia tarea para la adecuada adaptación de sus proyectos y planificaciones al grupo con el cual trabaje; mejorar las relaciones interpersonales y de trabajo grupal y consolidar habilidades para afrontar cambios.

La aplicación y práctica de competencias docentes tiene como objetivos:

1). Que el docente se sitúe frente sus propias prácticas en actitud reflexiva, pues en el espacio que ocupan las competencias cabe la definición exitosa de su práctica profesional en una relación proporcional.

2). Que el docente analice los supuestos que subyacen en su tarea pedagógica en relación con lo que enseña, cómo y para qué enseña, cómo aprenden sus estudiantes y cómo propicia la relación del contexto en el que enseña con el conocimiento en su trabajo cotidiano.

3). Que el docente cuente con la capacidad de tomar distancia para trabajar en función a sus capacidades y sus límites, analizarlas como si hubiesen sido realizadas por otro, evaluando de manera cotidiana las ventajas y desventajas de cada acción, brindándose a sí mismo (a) la posibilidad de revisar y ofrecer propuestas en caso de que se requiera. 


\subsection{La Didáctica: definición y caracterización como competencia docente} en Educación Inicial

La didáctica, según De La Torre, Oliver y Sevillano (2015): "es el arte de enseñar pedagogía según lo técnico y lo concreto; la parte que se ocupa de los sistemas y métodos prácticos de enseñanza destinados a plasmar en la realidad las directrices de la teoría pedagógica" (pág. 13). Se entiende entonces como una ciencia derivada de la pedagogía que realiza aportes al campo práctico de la enseñanza de maneras diversas y a partir de conocimientos y principios teóricos específicos, así como modelos, reglas, prácticas, métodos y estrategias variadas articuladas entre sí.

Es importante señalar en este punto que la didáctica no contempla sólo la enseñanza como versión y objeto de estudio, sino que éste es definido de diferentes modos. Concretamente en la Educación Inicial, la docente debe comprender que la didáctica tiene como desafío ayudarle a impartir conocimientos a sus estudiantes en consideración al contexto y a las diferencias socio-culturales, y que en esta interacción podrá trabajar en torno a la competencia intelectual, objetivo básico de todo proceso de enseñanza. Este nivel de exigencia profesional requiere de la docente una intersección permanente de la teoría con la práctica, comparando constantemente su formación con la realidad de su aula.

Sin embargo, en el Subsistema de Educación Inicial venezolano es común observar que, cotidianamente, las docentes no basan sus prácticas en principios didácticos (en el mejor de los casos lo hace en base a recursos lúdicos, no didácticos). Ello contribuye al franco deterioro de las competencias didácticas dentro del aula, pues la docente de Educación Inicial no sólo ha de ser "facilitadora" de conocimientos, sino que debe de hecho intervenir en ese proceso siendo mediadora, propiciar el aprendizaje. Por otra parte, debe dar importancia a los contenidos planteados curricularmente y entender la finalidad con la que éstos fueron concebidos teniendo en cuenta los sujetos de 
aprendizaje, los contextos, la articulación de teorías, la relación de dicha teoría con la práctica y la reflexión de su propia praxis. Esto permite caracterizar el objeto de la didáctica respecto a la enseñanza de la Historia y la cultura de la siguiente manera:

a). Que la docente internalice que, independientemente del nivel educativo, el abordaje de la didáctica como competencia no varía, más sí el contexto socio-cultural donde se le aplica.

b). Que la docente reflexione sobre los marcos teóricos instituidos relativos a prácticas pedagógicas con el fin de realimentar sus técnicas y estrategias según los logros obtenidos o dificultades presentadas.

c). Que la docente investigue y se documente eficientemente sobre el personaje o hecho histórico-cultural que desea que los niños y niñas conozcan, desmitificando el supuesto de que, dado que son muy pequeños, no es necesario que sepan temáticas de esta índole en profundidad.

Ahora bien, ¿qué implica la competencia docente de diseñar estrategias didácticas para enseñar Historia y cultura en el aula Inicial?. Al pretender enseñar Historia y cultura conscientemente a niños y niñas en el nivel Inicial se debe propiciar la reflexión crítica, abordando didácticamente temas que hagan referencia a hechos, personajes, lugares y objetos socioculturales significativamente históricos. Dicha reflexión va a generarse, en parte fundamental, en el desempeño que tenga la docente como mediadora del aprendizaje.

Para contribuir a la mejora procedimental de la enseñanza de la Historia y la cultura en la Etapa Preescolar en el Subsistema de Educación Inicial Bolivariano; la docente deberá evitar la incorporación de información supuestamente adaptada para que los niños y las niñas entiendan, pues desvirtúan su verdadero sentido. Debe asimismo manifestar intencionalidad respecto a informarse sobre un hecho histórico, cultural o un personaje 
importante; entendiendo que el enseñar nociones de Historia y cultura no implica reducir o desconocer el papel didáctico de éstas inherente a la tarea pedagógica de su nivel de enseñanza, el cual generalmente está reservado sólo para nociones lingüísticas y numéricas. En este mismo orden de ideas, no debe olvidar que el aprendizaje se distingue por ser interno e idiosincrásico, por lo que cada niño y niña deberá hacer su propia contribución, según sus características personales del aprendizaje ocurrido.

2.3. Análisis de teorías del aprendizaje: aportes a la didáctica de la enseñanza de la Historia y la cultura en Educación Inicial

a). Teoría: Psicogenética de Piaget (1969): Asimilación y acomodación y sistema de significantes. $1^{\circ}$ principio: La información $\mathrm{O}$ nociones de Historia y la cultura que el niño y la niña reciban deben ser adecuadas a su edad y compatible con su nivel de pensamiento, de otro modo carece de sentido y contextualización y, por lo tanto, no será internalizada. $\mathbf{2}^{\circ}$ principio: Las situaciones de aprendizaje de la Historia y la cultura tienen que surgir dentro del campo de la experiencia del niño y la niña, para que sus estructuras mentales cuenten con esquemas previos y puedan asimilar conocimientos en correspondencia a su edad. Aporte didáctico: Desarrollo de modelos de pensamiento intuitivo en reemplazo del pensamiento lógico.

b). Teoría: Socio-histórica de Vigotsky (1934): Aprendizaje significativo e interiorización. $3^{\circ}$ principio: El aprendizaje de la Historia y la cultura se convierte en apropiación de la cultura por el niño y la niña como sujetos que aprenden, en atención a la producción y reproducción del conocimiento, teniendo como base la orientación e interacción social. $4^{\circ}$ principio: Las situaciones de aprendizaje de la Historia y la cultura en Educación Inicial deben estar encaminadas al fomento de la personalidad moral del niño y la niña, como complemento a su desarrollo integral. Aporte didáctico: Interiorización de instrumentos culturales para el intercambio social 
con los adultos que les rodean; fomento del desarrollo moral a la par de los conocimientos prácticos que da lugar a la formación de orientaciones valorativas.

c). Teoría: Andamiaje de Bruner (1988): Aprendizaje por descubrimiento, mediación y construcción de estructuras mentales.

principio: La enseñanza de la Historia y la cultura es un proceso regresivo que debe abordarse no como un conocimiento adicional, sino como una reflexión permanente, favoreciendo la curiosidad básica en el niño y la niña que los lleve a preguntarse sobre el presente, el pasado y, por asociación, el futuro. $6^{\circ}$ principio: El descubrimiento de la abstracción histórico-cultural va a depender esencialmente de la mediación de la docente y la manera como ésta presente didácticamente el conocimiento a los niños y niñas durante el desarrollo de la actividad. Aporte didáctico: Construir realidades a partir de los estímulos que reciben, un mundo sobre la base de intenciones y significados, de esta manera el conocimiento no tiene un origen externo, sino que ha sido construido por ellos mismos, facilitando su significación y afianzamiento.

\subsection{Importancia de conocer sobre Historia y cultura en Educación Inicial: aportes desde el Currículo de Educación Inicial Bolivariano (CEIB)}

La enseñanza de la Historia y la cultura en Educación Inicial en Venezuela, puede determinarse en dos (02) etapas: la primera, desde los comienzos de la entonces llamada educación preescolar, siguiendo lo establecido por el Ministerio de Educación (1986): en el documento Guía Práctica de Actividades para Niños Preescolares, los cuales se ocupaban de mencionar la historicidad y la cultura de forma pasiva y lineal, meramente conductista, haciendo referencia pasajera a fechas y eventos sin mediación didáctica alguna: ocasiones como el Natalicio de Simón Bolívar, Día de la Bandera, Día de la Raza, Batalla de Carabobo y fechas como el 5 de julio, y el 
19 de abril, eran si acaso, mencionados sin programar actividades significativas dentro de la planificación, con el argumento de que a esa edad los niños no pueden entender temáticas de tal profundidad.

La segunda se ubica desde el advenimiento de la V República, en el documento sancionado por el Ministerio de Educación y Deportes (2005): denominado Educación Inicial: Bases curriculares en el cual surge, aparte del cambio de la denominación de preescolar a educación inicial: "[...] tiene como centro al ser humano como ser social, capaz de responder y participar activamente en la transformación de la sociedad en la que vive y se desarrolla" (pág. 5); por lo cual se asume la proposición del patriotismo como un sentimiento nacido de una comunidad de ideales y esperanzas, que debe estimularse en el espíritu comprensivo y flexible de los niños y las niñas sobre la visión de su nacionalidad. Este documento estableció, entre otras cosas, que la construcción curricular bolivariana está fundamentada en una visión humanista social. Esto se hizo más evidente en la redacción del documento Subsistema de Educación Inicial Bolivariana: Currículo y Orientaciones Metodológicas, emitido por el denominado Ministerio del Poder Popular para la Educación (2007b), donde el componente Historia local, regional y nacional, se menciona como meta formativa:

[...] Iniciar al niño y la niña en el conocimiento, respeto y valoración de su historia personal, familiar y social a nivel regional y nacional; así como en la toma de conciencia de su identificación con el entorno histórico-cultural y productivo [...]" (pág. 28).

En otras palabras, las particularidades de los niños y niñas en un ambiente que les provea de actividades, experiencias de vida humana y de aprendizajes, dan lugar al desarrollo. Señala también una serie de aprendizajes a ser alcanzados en relación al objeto de conocimiento, los cuales incluyen el reconocimiento de ciertas manifestaciones culturales del 
país y de su comunidad, así como hechos y personajes históricos sobresalientes del país y la comunidad.

De igual manera, abordan la práctica progresiva del respeto, la solidaridad, tolerancia, cooperación, honestidad y responsabilidad; también el reconocimiento del contexto local, lugares y edificaciones históricas y culturales: plazas, bibliotecas, museos; Identifica y valora los símbolos patrios, nacionales y regionales. Todo lo anterior tiene como finalidad el transferir, depositar, trasmitir valores y conocimientos teniendo como base un sólido argumento: propiciar la aparición y permanencia del sentido de pertinencia que debe ser expresado por cada uno, en el futuro, como ciudadanos y ciudadanas respecto a su lugar de origen.

\section{Metodología}

La investigación tuvo lugar en cinco (05) Centros de Educación Inicial de la ciudad de Barinas estado Barinas por espacio de cuatro (04) meses, desde enero hasta mayo del año 2019. Se aplicó instrumento de recolección de datos en la modalidad encuesta a diez (10) docentes pertenecientes a estos centros educativos, a razón de dos (02) docentes por cada uno (una docente de aula y la docente en función de Coordinadora de Sociedades Bolivarianas e Interculturalidad). La línea de investigación respondió al paradigma positivista con enfoque cuantitativo, utilizando un proceso inductivo para explorar y describir, lo cual generó perspectivas teóricas. En relación al tipo de la investigación, Balestrini (2006), afirma que la misma: "[...] está en función del tipo de problema que se pretenda estudiar y de los objetivos establecidos al interior del mismo [...]" (pág. 11). Siguiendo este criterio, este estudio se asume como de tipo descriptivo por la manera en que se realizó la búsqueda de la información en la fuente viva y primaria, por lo tanto, el análisis sistemático del problema en su realidad sirvió para describirlo, interpretarlo, entender su naturaleza y factores constituyentes. 
En relación al modelo, el presente estudio se presentó bajo la modalidad de proyecto factible, el cual según la Universidad Pedagógica Experimental Libertador (2016): "[...] consiste en la investigación, elaboración y desarrollo de una propuesta de un modelo operativo viable para solucionar problemas, requerimientos o necesidades de organizaciones o grupos sociales; puede referirse a la formulación de políticas, programas, tecnologías, métodos 0 procesos [...]" (pág. 21). Resulta entonces el medio más eficaz para promover estrategias que van a permitir el surgimiento de las soluciones hacia la resolución de la problemática detectada en el presente estudio, a partir de lo cual se pudo caracterizar la situación. Las variables fueron consideradas en atención a los objetivos del estudio, siendo la variable independiente el desarrollo de competencias docentes y la dependiente la enseñanza de nociones histórico-culturales.

Para la obtención de resultados del presente estudio, la recolección de datos se organizó a partir de las técnicas de observación directa y encuesta, utilizando para ello un instrumento tipo cuestionario con un total de diez (10) interrogantes con cuatro (04) opciones de respuesta tomando en consideración el posicionamiento en escala de Likert. Este instrumento fue validado mediante la técnica de juicio de expertos y una prueba piloto para medir la confiablidad. Luego se procedió a la representación estadística de los mismos a partir del modelo de gráfico circular, utilizando para su elaboración el software computarizado para hojas de cálculo, Microsoft Excel.

\section{Resultados}

La presente investigación se realizó con el propósito de indagar acerca del uso y la aplicación de medios didácticos docentes para la enseñanza de la Historia y la cultura en cinco (05) Centros de Educación Inicial en la ciudad de Barinas estado Barinas. Posterior a la aplicación de instrumento, se obtuvieron los siguientes datos porcentuales, mencionados en la tabla 1. 
Tabla 1. Instrumento de recolección de datos y porcentaje de respuestas obtenidas.

\begin{tabular}{|c|c|c|c|c|c|}
\hline \multirow[b]{2}{*}{ N. ${ }^{\circ}$} & \multirow[b]{2}{*}{ ÍTEMS } & \multicolumn{4}{|c|}{ OPCIONES } \\
\hline & & $\frac{\underset{c}{W}}{\frac{w}{\alpha}}$ & 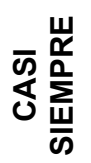 & 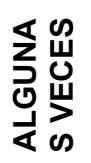 & $\begin{array}{l}\overleftarrow{U} \\
\text { Z } \\
\mathbf{z}\end{array}$ \\
\hline 01 & $\begin{array}{l}\text { ¿Realiza usted diariamente mediación significativa } \\
\text { sobre hechos de Historia y cultura, así como de } \\
\text { personajes históricos locales, regionales y } \\
\text { nacionales con sus estudiantes? }\end{array}$ & - & $12 \%$ & $20 \%$ & $68 \%$ \\
\hline 02 & $\begin{array}{l}\text { ¿Planifica usted con la Coordinadora de } \\
\text { Sociedades Bolivarianas y otras colegas } \\
\text { actividades de visita a sitios históricos y de } \\
\text { relevancia cultural de la comunidad, el estado o el } \\
\text { país? }\end{array}$ & - & - & - & $100 \%$ \\
\hline 03 & $\begin{array}{l}\text { ¿Organiza usted eventos colectivos que incluyan a } \\
\text { los representantes y otros miembros de la } \\
\text { Comunidad Educativa en el aula para evaluar } \\
\text { aprendizajes sobre hechos y personajes } \\
\text { relacionados a la historia o cultura local, regional y } \\
\text { nacional? }\end{array}$ & - & - & - & $100 \%$ \\
\hline 04 & $\begin{array}{l}\text { ¿Incluye usted en sus indicadores de evaluación } \\
\text { diaria competencias relacionadas al fomento y } \\
\text { valoración de la histórica y la cultura local, regional } \\
\text { y nacional? }\end{array}$ & - & - & $30 \%$ & $70 \%$ \\
\hline 05 & $\begin{array}{l}\text { ¿Procura usted formarse continuamente, leyendo } \\
\text { e investigando respecto a hechos y personajes } \\
\text { relacionados a la historia o cultura local, regional y } \\
\text { nacional? }\end{array}$ & - & $12 \%$ & $20 \%$ & $68 \%$ \\
\hline 06 & $\begin{array}{l}\text { ¿Incluye usted en su planificación semanal al } \\
\text { menos una actividad colectiva relacionada con la } \\
\text { valoración de hechos y personajes relacionados a } \\
\text { la historia o cultura local, regional y nacional? }\end{array}$ & - & - & $20 \%$ & $80 \%$ \\
\hline 08 & $\begin{array}{l}\text { ¿Considera usted que el fomento de la historia y la } \\
\text { cultura en el Centro de Educación Inicial está } \\
\text { relacionada a proselitismo político o imposición de } \\
\text { ideologías? }\end{array}$ & - & $40 \%$ & $60 \%$ & - \\
\hline 09 & $\begin{array}{l}\text { ¿Presenta usted diariamente a sus estudiantes } \\
\text { material didáctico asociado a la valoración de } \\
\text { hechos y personajes relacionados a la historia o } \\
\text { cultura local, regional y nacional? }\end{array}$ & - & $20 \%$ & $10 \%$ & $70 \%$ \\
\hline 10 & $\begin{array}{l}\text { ¿Piensa usted que es útil contar con una guía } \\
\text { didáctica que le oriente estratégicamente sobre } \\
\text { actividades dirigidas a fomentar la conciencia } \\
\text { histórica y cultural en sus estudiantes? }\end{array}$ & $100 \%$ & - & - & - \\
\hline
\end{tabular}

Fuente: La Autora (2019). 
El análisis de los datos obtenidos a partir de las respuestas, permite concluir el hallazgo de evidencias concretas que permiten perfilar la situación problema, a saber:

- Ausencia de mediación didáctica desde la perspectiva de la intervención docente (planificación, estrategias) y el uso de recursos instruccionales adecuados al nivel.

- Ausencia de instrumento e indicadores de evaluación específicos para actividades de índole histórico-cultural.

A razón de las evidencias obtenidas, se establecen las siguientes necesidades:

- Perfeccionar el conocimiento de la Historia y la cultura local, regional y nacional por parte de las docentes.

- Adecuar el uso de recursos didácticos e instruccionales para la enseñanza de la historia y la cultura en los Centros de Educación Inicial (CEI) de la ciudad de Barinas, estado Barinas.

- Promover la adquisición de conocimiento de hechos y personajes histórico-culturales en los niños y niñas a partir del sentimiento de cambio, la idea de duración, la reconstrucción de hechos y el sentimiento de empatía hacia la historia y la cultura de nuestro país.

- Reconocer las formas en que el niño y la niña construyen su conocimiento.

- Reflexionar sobre la práctica docente relacionada con la enseñanza de la Historia y la cultura en los Centros de Educación Inicial (CEI).

- Mejorar y complementar continuamente, a partir de la investigación, sus conocimientos en Historia local, regional y nacional, así como en cultura y valores tradicionales.

- Diseñar una guía didáctica para el desarrollo de nociones histórico- 
culturales en los niños y niñas en Etapa Preescolar como apoyo al currículo, basada en tres (03) principios: 1). El tiempo se mide, pasa y cambia de forma constante 2). Las cosas no siempre han estado en el sitio actual ni han sido iguales 3 ). El niño y la niña deben reflexionar acerca de cómo sería nuestra vida sin las cosas, lugares y personas que hoy existen.

\section{Conclusiones}

Cuando un docente inicia la búsqueda del cumplimiento del proceso enseñanza-aprendizaje, busca generar una praxis-acción renovada y transformadora en la que intervenga todo el contexto, siguiendo a ello una continua reflexión. La intencionalidad de la autora es generar una solución a la problemática detectada, por lo cual se establece como proyecto factible la redacción de una propuesta de guía didáctica como material de apoyo docente, cuya elaboración pretende beneficiar en forma consciente y sistematizada el perfeccionamiento de competencias -atendiendo a un sentido social reflexivo, crítico e innovador- para la enseñanza de la Historia y la cultura en la Etapa Preescolar, siendo la mediación de la docente el elemento fundamental para lograr que éstos vayan pasando a niveles progresivos de pensamiento cada vez más complejos, favoreciendo con ello la construcción del pensamiento histórico.

Esto les permitirá a las docentes crear herramientas con las cuales sus niños y las niñas puedan acceder efectivamente al conocimiento de la Historia y la cultura, a partir de experiencias diversas que les generen curiosidad y el deseo de indagar en el pasado de tal manera que les sea significativo, constituyendo un referente ideológico para el fomento de su desarrollo moral. Dicha guía creará asimismo la alternativa para la generación de estrategias didácticas que permitan innovar la práctica docente teniendo en cuenta lo teórico, lo metodológico y lo instrumental. 
Esta propuesta busca relacionar la didáctica con el aprendizaje de nociones de tiempo y espacio, a partir de la premisa de que el pasado puede ser reconstruido para su comprensión y que aprender historia y cultura es una experiencia agradable. Los resultados obtenidos pueden servir de base para orientar a quien manifieste la misma inquietud científica, con la perspectiva de llegar a servir de modelo a futuras investigaciones encaminadas a la modificación del Currículo de Educación Inicial Bolivariano.

\section{Referencias}

Balestrini, M. (2006). Cómo se elabora el Proyecto de Investigación. $7 \mathrm{ma}$ edición, ISBN: 980-6293-03-7. Caracas, Venezuela: BL Consultores Asociados.

Bruner, J. (1988). Realidad mental y mundos posibles. Los actos de la imaginación que dan sentido a la experiencia. Trad. de Beatriz López. Barcelona, España: Editorial Gedisa.

Coronado, M. (2013). Competencias Docentes: ampliación, enriquecimiento y consolidación de la práctica profesional. 2da Edición, Colección Educación y Trabajo, ISBN: 9789875382596. Buenos Aires, Argentina: Editorial Novedades Educativas, NOVEDUC.

De La Torre, S., Oliver, C., \& Sevillano, M. (2015). Estrategias didácticas en el aula: buscando la calidad y la innovación. Barcelona, España: Narcea Ediciones.

García, F. (2015). La investigación en didáctica de las Ciencias Sociales y la construcción del conocimiento profesional de los docentes. México, D.F.: Editorial Santos Ávila Ruíz.

Ministerio de Educación y Deportes (2005). Educación Inicial: Bases curriculares. Depósito legal: If 68420043753598. Caracas, Venezuela: Editorial Noriega.

Ministerio de Educación (1986). Guía Práctica de Actividades para Niños 
preescolares. Tomos I y II. Caracas, Venezuela: Editorial Sauapi. Ministerio del Poder Popular para la Educación (2007a,b). Subsistema de Educación Inicial Bolivariana: Currículo y Orientaciones Metodológicas. ISBN: 978-980-218-282-4. Caracas, Venezuela: Fundación Centro Nacional para el Mejoramiento de la Enseñanza de Ciencia, CENAMEC.

Piaget, J. (1969). Psicología del Niño. Reeditado por Bärbel Inhelder, 21ava Edición, 2015. Madrid, España: Ediciones Morata.

Salto, M., \& Cendón, A. (2019). Incidencia del Desempeño Profesional del Docente de Educación Inicial. Revista Scientific, 4(13), 160-181, eISSN: 2542-2987. Recuperado de:

https://doi.org/10.29394/Scientific.issn.2542-2987.2019.4.13.8.160-181 Universidad Pedagógica Experimental Libertador (2016). Manual de Trabajos de Grado de Especialización y Maestría y Tesis Doctorales. 5ta Edición, ISBN: 980-273-441-1. Caracas, Venezuela: Fondo Editorial de la Universidad Pedagógica Experimental Libertador, FEDUPEL.

Vigotsky, L. (1934). La teoría socio-histórica y el desarrollo de procesos psicológicos superiores. 24ava reedición. México, D.F.: Editorial Grijalbo. 
OAI-PMH: http://www.indteca.com/ojs/index.php/Revista_Scientific/oai

Artículo Original / Original Article

\section{Laury Dayana Mejías Escorcha}

e-mail: laurymejias84@gmail.com

Nacida en la localidad de Sabaneta, estado Barinas, el 4

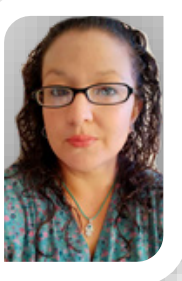
de marzo del año 1984. Docente ordinaria en condición de instructora a tiempo completo en la Universidad Pedagógica Experimental Libertador, Instituto de Mejoramiento Profesional del Magisterio (UPEL-IMPM), Barinas, estado Barinas; Egresada de esta misma institución en julio del año 2017, como Profesora de Educación Preescolar; He cumplido labores como docente de aula y Coordinadora Pedagógica adscrita al Ministerio del Poder Popular para la Educación en el Subsistema de Educación Inicial en los municipios Alberto Arvelo Torrealba de Barinas, en Bolívar del estado Barinas y el municipio Libertador del estado Mérida; Actualmente estoy adscrita al Ministerio del Poder Popular para la Educación Universitaria, ejerzo labores de apoyo docente y logística administrativa en la Coordinación de Secretaría y Registro Estudiantil en la Universidad Pedagógica Experimental Libertador (UPEL-IMPM), sede Barinas, estado Barinas.

El contenido de este manuscrito se difunde bajo una Licencia de Creative Commons ReconocimientoNoComercial-Compartirlgual 4.0 Internacional 